\title{
Etude des Impacts des Changements Climatiques sur les Activités Agricoles dans la Commune Rurale de Mafouné, Cercle de Tominian, Région de Ségou au Mali
}

Issa Ouattara, PhD

Docteur en Géographie de l'Environnement, Enseignant-Chercheur à l'Institut de Développement Economique et Social de Bamako, Mali

\section{Yakouréoun Diarra, $M A$}

Doctorant en Sociologie de l'Environnement à l'Institut de Pédagogie Universitaire de Bamako, Chercheur à l'Institut des Sciences Humaines de Bamako, Mali

\section{Seydou Mariko, PhD}

Maître de Conférences, Géographe, Professeur au Département d'Enseignement et de Recherche d'Histoire et de Géographie de l'Ecole Normale Supérieure de Bamako, Mali

Doi:10.19044/esj.2019.v15n11p121 URL:http://dx.doi.org/10.19044/esj.2019.v15n11p121

\section{Résumé}

L'agriculture est l'un des secteurs les plus vulnérables aux effets des changements climatiques. La présente étude a pour objectif d'analyser les perceptions et les impacts des changements climatiques sur les activités agricoles, mais aussi, de contribuer à une meilleure connaissance des stratégies d'adaptation dans la Commune Rurale de Mafouné au Mali. La méthodologie utilisée s'est appuyée sur la recherche documentaire, l'observation directe, les enquêtes de terrain auprès des agriculteurs et agro-pasteurs, des responsables des Organisations Non Gouvernementales, des structures techniques et l'analyse des données météorologiques. Les données collectées du 02 au 30 mai 2018 ont fait l'objet d'un traitement informatique à l'aide des logiciels SPSS 20.00 et Excel 2016 et d'une analyse de contenu des discours. Les résultats de l'étude ont montré une tendance pluviométrique à la baisse avec une très grande variabilité interannuelle et une hausse des températures. Ceci a comme impacts, la baisse des rendements agricoles, la perte de la fertilité des sols, le tarissement précoce des eaux de surface, l'endettement du monde paysan, l'intensification de l'exode rural. Le semis des variétés hâtives, la pratique des techniques de lutte anti-érosive, le reboisement, la diversification des activités sont entre autres des stratégies d'adaptation développées par les 
exploitants pour faire face aux effets néfastes des changements climatiques. L'étude a conclu qu'une meilleure maitrise des pratiques d'adaptation et l'accompagnement des exploitants sont de véritables palliatifs aux impacts des changements climatiques sur les activités agricoles dans la Commune Rurale de Mafouné.

Mots-clés : adaptation, changements climatiques, impacts, Mafouné

Study of the Impacts of Climate Change in the Agricultural Activities in the Rural Commune of Mafouné in the Area of Tominian, Ségou Region (Mali)

\title{
Issa Ouattara, PhD
}

Docteur en Géographie de l'Environnement, Enseignant-Chercheur à

l'Institut de Développement Economique et Social de Bamako, Mali

\section{Yakouréoun Diarra, $M A$}

Doctorant en Sociologie de l'Environnement à l'Institut de Pédagogie Universitaire de Bamako, Chercheur à l'Institut des Sciences Humaines de Bamako, Mali

Seydou Mariko, PhD

Maître de Conférences, Géographe, Professeur au Département d'Enseignement et de Recherche d'Histoire et de Géographie de l'Ecole Normale Supérieure de Bamako, Mali

\begin{abstract}
Agriculture is one of the most vulnerable sectors to the effects of climate change. The objective of this study is to analyse the perceptions and impacts of climate change on agricultural activities, but also to contribute to a better understanding of adaptation strategies in the Rural Commune of Mafouné in Mali. The methodology used relied on documentary research, direct observation, field surveys of farmers and agro-pastoralists, officials of non-governmental organizations, technical structures and analysis of meteorological data. The data collected from May, $2^{\text {nd }}$ to May $30^{\text {th }} 2018$ were processed using the SPSS 20.00 and Excel 2016 software and a speech content analysis. The results of the study showed a declining rainfall trend with very high interannual variability and rising temperatures. This has as impacts, the
\end{abstract}


fall of the agricultural yields, the loss of the soils fertility, the early drying up of the surface waters, the indebtedness of the peasant world, the intensification of the rural exodus. The seed of early varieties, the practice of erosion control techniques, reforestation, and diversification of activities are some of the strategies developed by farmers to cope with the negative effects of climate change. The study concluded that a better control of adaptation practices and support for farmers constitute the real palliative to the impacts of climate change on agricultural activities in the Rural Commune of Mafouné.

Keywords: Adaptation, climate change, impacts, Mafouné

\section{Introduction}

Le changement climatique, encore appelé réchauffement global, est un phénomène d'augmentation des températures moyennes des océans et de l'atmosphère au niveau planétaire. Les résultats d'évaluations scientifiques aussi bien mondiales que régionales $d u$ Groupe d'Experts Intergouvernemental sur l'Evolution du Climat (GIEC) confirment que le climat de la terre est en train de changer. Ce changement serait dû au réchauffement climatique, que les scientifiques imputent à l'émission de gaz à effet de serre, conséquence des activités de l'homme (GIEC, 2007). Ses caractéristiques et ses conséquences sont multiples à cerner tant à l'échelle planétaire, régionale que locale. La modification de la température, des précipitations et d'autres variables du climat, pourrait se traduire par des changements mondiaux dans l'humidité du sol, par une élévation du niveau moyen de la mer et par la perspective d'épisodes plus graves de fortes chaleurs, d'inondations, de sécheresses (N'diaye, 2015).

Il y a encore quelques années, le débat sur les changements climatiques était limité à un groupe de scientifiques, dont les théories étaient souvent qualifiées de surréalistes. Aujourd'hui, tout le monde en parle, car, il existe des preuves indiscutables qui confirment que la concentration élevée de gaz à effet de serre dans l'atmosphère entraine le réchauffement de la planète. D'ici la fin du XXI ${ }^{\text {ème }}$ siècle, il est prévu que la température moyenne au sol augmente de 1,5 à $6^{\circ} \mathrm{C}$. Ce réchauffement sera important à l'intérieur et dans les franges semi-arides du Sahara (AGRIDAPE, 2009). Les modifications que ces changements induiront dans l'intensité et la répartition des pluies qui rythment l'essentiel de la production africaine ne semblent pas encore bien maitrisées. Ce qui constitue un défi et un enjeu majeurs à l'échelle globale, régionale et locale pour les écosystèmes et pour les populations en raison des conséquences imprévisibles qu'ils engendrent comme la récurrence des évènements climatiques extrêmes. Dans son rapport d'évaluation de 2007, le Groupe d'Experts Intergouvernemental sur l'Evolution du Climat estime que la hausse globale des températures et la fréquence des évènements climatiques 
extrêmes ont comme incidences, les fortes canicules, les précipitations fortes ou déficitaires. Celles-ci impactent fortement sur les activités économiques et mettent en péril les moyens de subsistance des populations.

$\mathrm{Au}$ Sahel, plusieurs études estiment une évolution des isohyètes vers le Sud. Selon ces études, la distance parcourue par les isohyètes dans le sens Nord-Sud est de 200 à $300 \mathrm{~km}$ pour l'isohyète $100 \mathrm{~mm}, 100$ à $150 \mathrm{~km}$ pour l'isohyète $500 \mathrm{~mm}$ et $100 \mathrm{~km}$ pour l'isohyète $1000 \mathrm{~mm}$. Il s'en suit une dégradation des ressources naturelles et une fragilisation des systèmes de production. La destruction du couvert végétal, la surexploitation des terres agricoles et l'abandon ou la réduction de la durée des jachères ont conduit à la dégradation des sols, à la baisse des rendements agricoles et à l'insécurité alimentaire (Konaté, 2009 ; N'diaye, 2015).

Le Mali, vaste pays continental, est très vulnérable à la variabilité et au changement climatique à l'instar des autres pays sahéliens. Cette vulnérabilité est souvent due à la dépendance des populations rurales aux ressources naturelles. Environ, $75 \%$ de la population malienne sont des ruraux et dépendent des ressources naturelles pour leur subsistance (PANA, 2007) ${ }^{5}$. L'expansion démographique entraine une extension des zones agricoles qui conduit à l'exploitation des zones pastorales peu productives, et à la destruction d'importants habitats naturels. Ces facteurs, en plus des phénomènes climatiques causés par la variabilité et le changement climatique (l'érosion sur toutes ses formes, forte sécheresse, etc) représentent les causes principales de la dégradation des terres. Au Mali, les principales ressources naturelles qui sont : l'eau, les sols, la faune et la flore sont menacées par le phénomène de désertification qui se manifeste par la dégradation des terres agricoles, la disparition des espèces végétales et animales, l'ensablement des cours d'eau et la dégradation de la qualité des eaux. Le régime pluviométrique est très affecté par la variabilité et les changements climatiques du fait de la tendance à la baisse de la pluviosité (Sanogo, 2012). Cette situation a pour conséquence, le démarrage tardif de la saison des pluies avec des poches de sécheresse, la diminution de la durée de la saison pluvieuse ainsi que l'intensification de l'exode rural.

La Commune Rurale de Mafouné avec un climat tropical sec de type soudano-sahélien, est située dans le Cercle de Tominian, Région de Ségou au Mali. Avec une pluviométrie très variable oscillant entre 600 à $900 \mathrm{~mm}$ par an, la Commune n'est pas à l'abri des phénomènes climatiques extrêmes tels que : les sécheresses, les inondations, les vents violents qui impactent

\footnotetext{
${ }^{5}$ Programme d'Action National pour l'Adaptation aux Changements Climatiques. (2007), cité par Sanogo, M.K. (2012). Capitalisation des bonnes pratiques de gestion durable des terres pour l'adaptation à la variabilité et au changement climatique au Mali : analyse d'impacts agronomiques, environnementaux et socio-économiques, mémoire de Master, Centre Régional AGRHYMET, Niger, p.22.
} 
négativement les activités économiques et mettent en péril les moyens de subsistance des populations locales. Les principales activités économiques qui sont : l'agriculture, l'élevage, la pêche et la cueillette, sont toutes fortement menacées par le changement climatique. Face à cette situation, les agriculteurs et agro-pasteurs développent des stratégies qui sont la plupart des techniques endogènes. Ces savoirs et pratiques des communautés rurales permettraient à leurs détenteurs de résister dans une certaine mesure, aux effets néfastes du climat, bien avant l'intervention des pouvoirs publics ou des Organisations Non Gouvernementales telles que : Sahel Eco, Caritas-Mali, World Vision, Lux-Développement. C'est pourquoi, l'étude des changements climatiques sur les activités agricoles est d'une importance capitale. Le présent article vise donc à analyser les impacts des changements climatiques sur les activités agricoles dans la Commune Rurale de Mafouné afin de contribuer à une meilleure connaissance des stratégies d'adaptation.

\section{Matériel et méthodes}

\section{Présentation du site d'étude}

L'étude a été réalisée du 02 au 30 mai 2018 dans la Commune Rurale de Mafouné, Cercle de Tominian, Région de Ségou au Mali. Avec une superficie de $866 \mathrm{~km}^{2}$, la Commune Rurale de Mafouné compte une population de 31116 habitants, dont 15502 hommes et 15614 femmes (Service Local de la Statistique, de l'Informatique, de l'Aménagement du Territoire et de la Population de Tominian, 2017). Elle connait un climat tropical de type soudano-sahélien avec une mauvaise répartition de la pluie dans le temps et dans l'espace (figure 1).

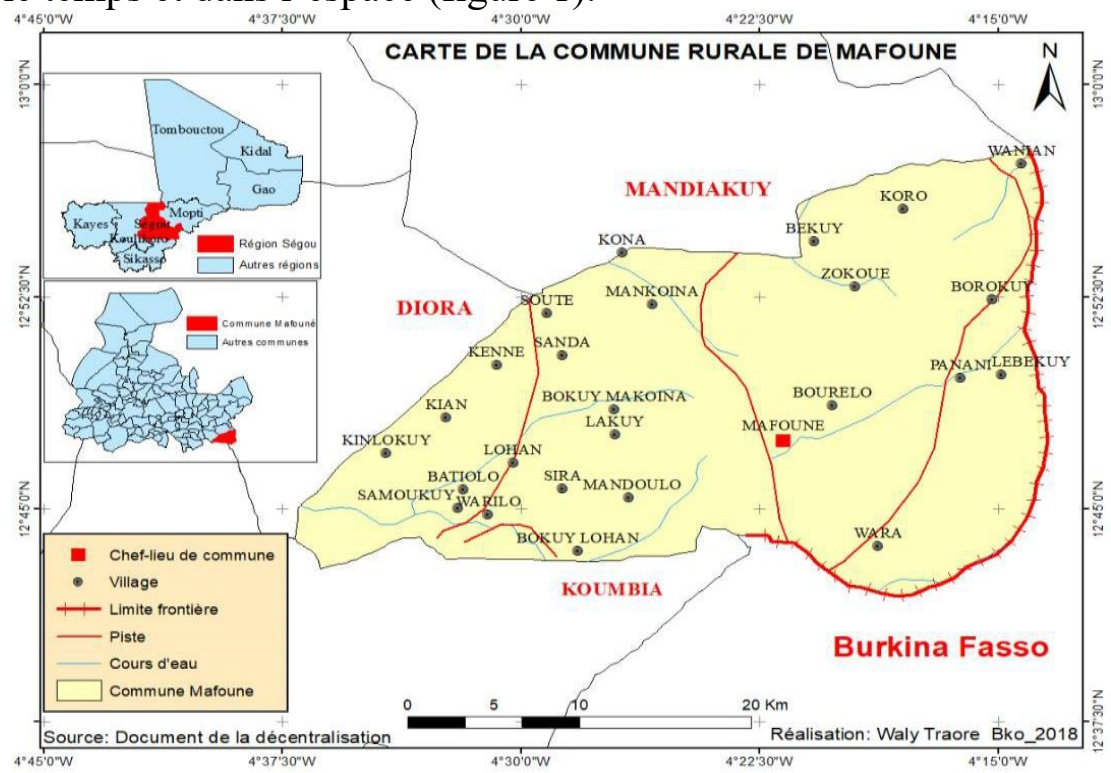

Figure 1 : Localisation de la Commune Rurale de Mafouné 


\section{Méthodes et outils}

L'approche méthodologique adoptée pour l'étude s'est appuyée sur la recherche documentaire, l'observation directe, la réalisation d'enquêtes quantitative et qualitative et l'analyse des données météorologiques. La recherche documentaire a consisté en la consultation et l'exploitation des ouvrages, des thèses, des mémoires universitaires, des rapports, des articles scientifiques en relation avec la thématique. Des sources Internet ont également été mises à profit. Ce travail nous a permis de mieux appréhender le sujet. L'observation directe a été menée par le biais de visites de terrain. Ces visites nous ont conduit dans les champs, les forêts, au niveau des différents points d'eau afin d'avoir une meilleure compréhension des techniques et des stratégies développées par les acteurs sur le terrain.

A partir de la liste des exploitants agricoles exploitée auprès du service d'agriculture de la Commune, un échantillon de 100 exploitants a été retenu. Les 100 personnes touchées par l'enquête quantitative étaient composées de 80 hommes et 20 femmes. La différence entre les hommes et les femmes s'explique d'une part par le rôle prépondérant des exploitations familiales où les chefs de ménages ont été majoritairement enquêtés et d'autre part, par l'appartenance des parcelles. Pour des raisons de connaissances historiques de l'évolution et des caractéristiques climatiques, les exploitants âgés de 30 ans au moins, ont été enquêtés. Sur le plan professionnel, la population enquêtée comprenait 75 agriculteurs et 25 agro-éleveurs.

La Commune Rurale de Mafouné compte vingt-neuf villages. Un tirage aléatoire simple nous a permis de retenir et d'enquêter dans sept villages. Il s'agit des villages de: Mafouné, Mankoina, Lohan, Mouni, Mandoulo, Sira et Lakuy. Le questionnaire adressé aux agriculteurs et aux agro-pasteurs de ces villages était relatif à leur connaissance et à leur perception sur les changements climatiques, aux impacts des changements climatiques sur les activités agricoles et aux stratégies d'adaptation aux changements climatiques. Sept focus group (dont un par village enquêté) ont également permis de compléter et de conforter les données quantitatives. Ces focus group ont concerné les exploitants agricoles. En ce qui concerne l'enquête qualitative, elle a été réalisée auprès de dix personnes ressources à travers un guide d'entretien. Ces personnes ressources étaient des responsables des services techniques d'agriculture de la Commune Rurale de Mafouné et du Cercle de Tominian, de la mairie de la Commune, des Organisations Non Gouvernementales intervenant dans le domaine du développement rural et de l'environnement dans la zone d'étude. Ces personnes ressources ont été sélectionnées, en fonction de leurs responsabilités et leur solide connaissance du sujet. Le type d'entretien utilisé a été l'entretien libre ou non directif, qui se caractérise par des questions non formulées d'avance dont les thèmes seulement sont précis. Les thématiques abordées lors des différents entretiens 
portaient sur la perception et la connaissance des changements climatiques, leurs impacts et les stratégies d'adaptation.

Quant aux données météorologiques, c'est-à-dire les données statistiques relatives aux températures et à la pluviométrie de la zone d'étude, elles ont été recueillies auprès de la Direction Nationale de la Météorologie du Mali. Les données quantitatives recueillies sur le terrain ont été traitées à l'aide des logiciels SPSS 20.00 et Excel 2016. Elles nous ont permis de traduire les résultats sous forme de tableaux et de graphiques. Par contre, les données qualitatives ont fait l'objet d'une analyse de contenu des discours. Cette analyse a consisté à rendre compte de ce qu'ont dit les interviewés de la façon la plus objective possible et la plus fiable possible. Pour ce faire, nous avons procédé à la transformation des discours oraux des enquêtés en texte. Ensuite, les informations similaires et différentes entre les répondants ont été interprétées.

\section{Résultats et Discussion}

\section{Perceptions paysannes sur les changements climatiques}

Les perceptions des exploitants sur les changements climatiques dans la zone d'étude portent sur l'évolution globale des saisons de pluies, de la température et des évènements climatiques extrêmes. Ces résultats sont représentés sur les figures suivantes.

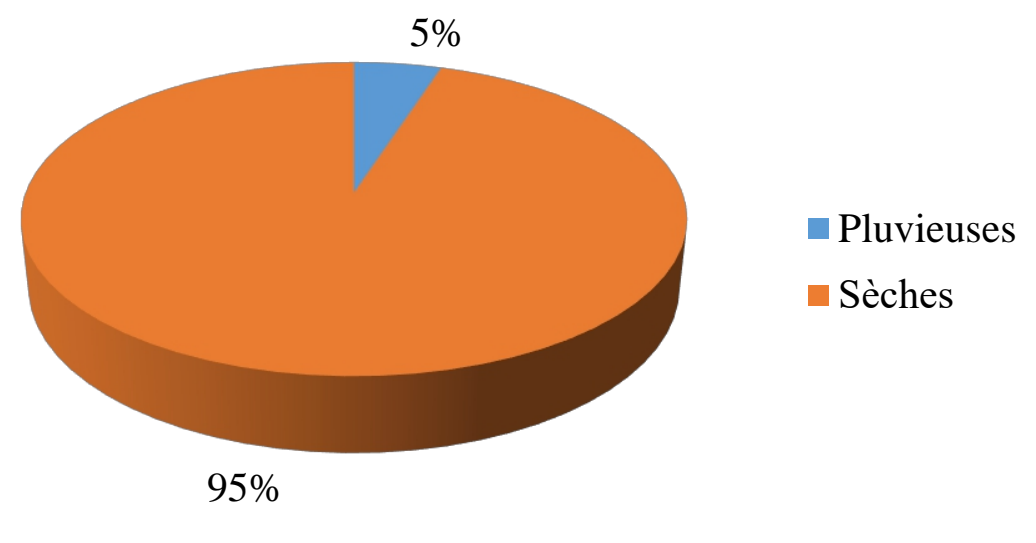

Figure 2 : appréciation des enquêtés sur l'évolution globale des saisons de pluies

Les résultats de l'étude montrent que les saisons de pluies deviennent de plus en plus sèches au cours des dernières années, selon la quasi-totalité des exploitants enquêtés. Certains enquêtés ont également laissé entendre que les saisons de pluies sont de plus en plus variables. Cette variabilité a des impacts considérables sur les activités agricoles de la Commune, tant sur la répartition, que sur la disponibilité de l'eau. Les précipitations (hauteur, durée de la saison de pluies, fréquence des pluies dans le temps et dans l'espace), les 
températures maximale et minimale sont les facteurs climatiques déterminant dans les indicateurs des changements climatiques. Compte-tenu de cette situation, il est important de mettre en relation les indicateurs des changements climatiques et les perceptions des enquêtés.

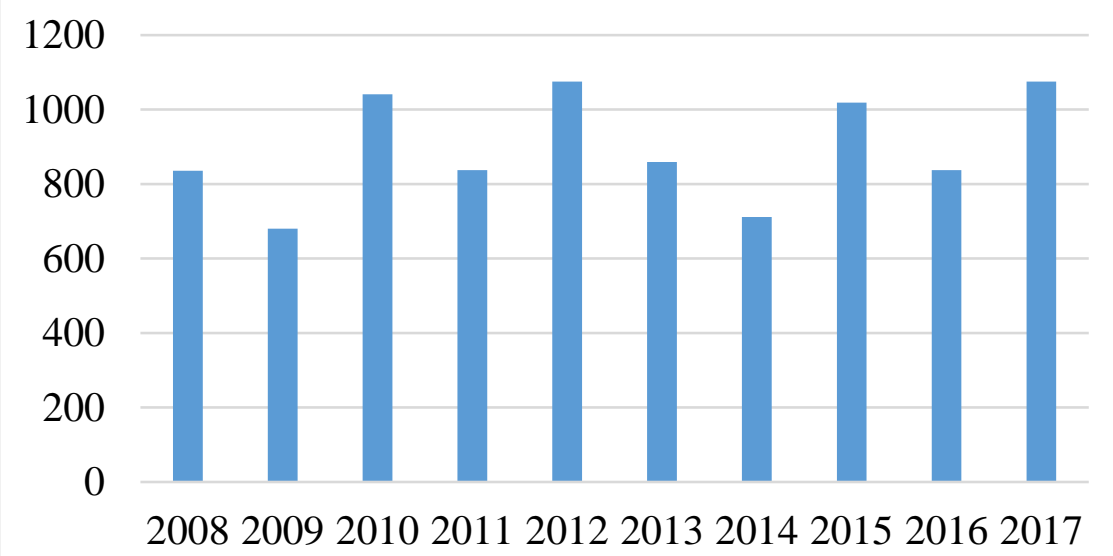

Figure 3 : évolution des précipitations interannuelles enregistrées à Mafouné, de 2008 à 2017

Source : Secteur Agriculture de Tominian, 2017

L'analyse des données sur les précipitations interannuelles enregistrées à Mafouné montre que cette Commune est suffisamment arrosée. La moyenne interannuelle des hauteurs d'eau enregistrée à la station est de $897,15 \mathrm{~mm}$ sur les dix (10) ans. On constate également une variation interannuelle très lisible de la pluviométrie avec une hauteur pluviométrique maximale annuelle de $1075 \mathrm{~mm}$ enregistrée en 2012 et 2017. La hauteur pluviométrique minimale est de 680,5 mm enregistrée en 2009. Cependant, malgré les moyennes pluviométriques assez élevées, la Commune Rurale de Mafouné n'est pas exempte des effets négatifs du réchauffement climatique. En 2017, avec une pluviométrie de $1075 \mathrm{~mm}$, la Commune est confrontée à un déficit céréalier dû à une mauvaise récolte. Ce mécanisme est dû à la répartition spatio-temporelle des précipitations qui se manifestent par le retard dans l'installation de la saison des pluies, l'arrêt précoce de la pluie, les pluies torrentielles aboutissant parfois à des inondations, l'accentuation du phénomène de l'érosion hydrique, mais aussi des poches de sécheresses successives durant la saison pluvieuse. Les moyennes pluviométriques enregistrées pendant ces dix dernières années ne doivent pas occulter les réalités climatiques de la zone. Pour mieux caractériser les manifestations des changements climatiques, nous avons jugé utile d'analyser les données pluviométriques interannuelles enregistrées au niveau du Cercle de Tominian (figure 4). 


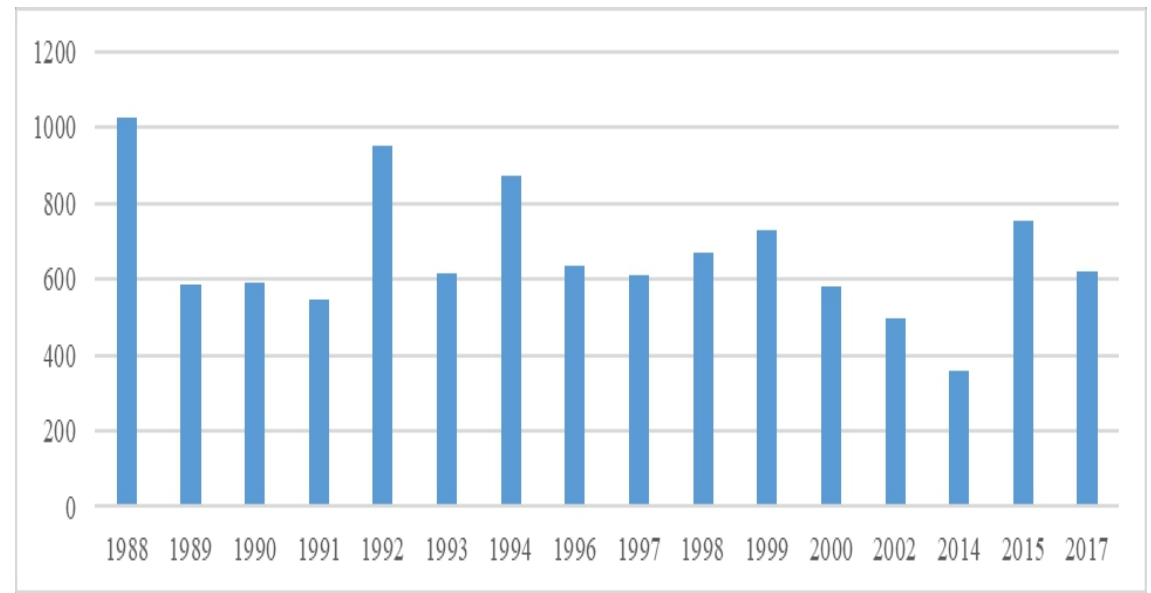

Figure 4 : évolution des précipitations interannuelles dans le Cercle de Tominian, de 1988 à 2017

Source : Météo-Mali, 2017

L'analyse de la figure 4 ci-dessus montre une tendance pluviométrique à la baisse. De façon générale, depuis 1988 où le Cercle a enregistré une pluviométrie annuelle de plus de $1000 \mathrm{~mm}$, on constate une tendance à la baisse, mais aussi, avec une variation de la pluviométrie d'année en année. L'année la plus sèche enregistrée ces trente (30) dernières années fut 2014, où une pluviométrie de $357,3 \mathrm{~mm}$ a été enregistrée dans le Cercle. Cette baisse de la pluviométrie constatée ces trois (3) dernières décennies et la variabilité pluviométrique interannuelle montrent, en effet que la zone subit les effets des changements climatiques. Les conséquences de la diminution de la pluviométrie ont des impacts considérables sur les activités agricoles, sources de revenu des populations de la zone.

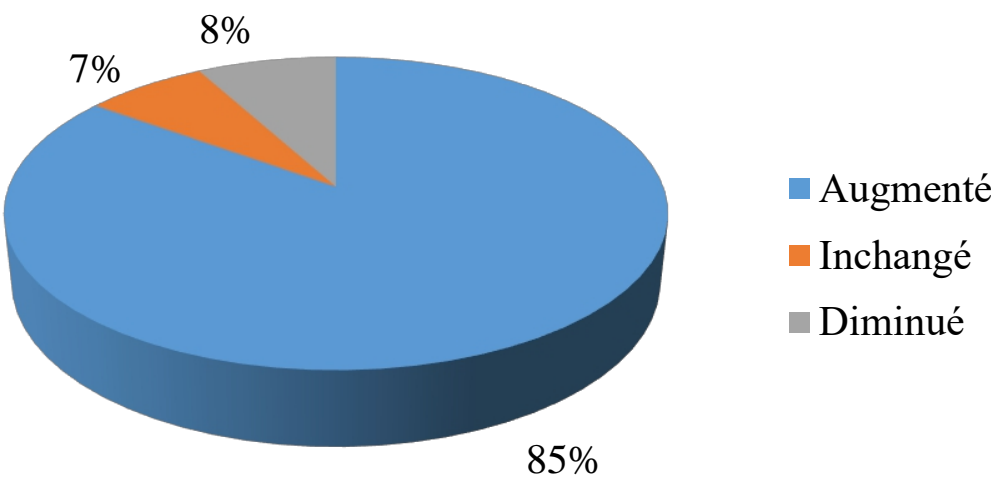

Figure 5 : perception paysanne sur l'évolution globale de la température 
La figure 5 indique que l'écrasante majorité des enquêtés perçoivent l'augmentation de la température. Les enquêtés font référence à ce titre, à l'intensité du soleil, à la fréquence de fortes canicules et les besoins de plus en plus croissants en eau.

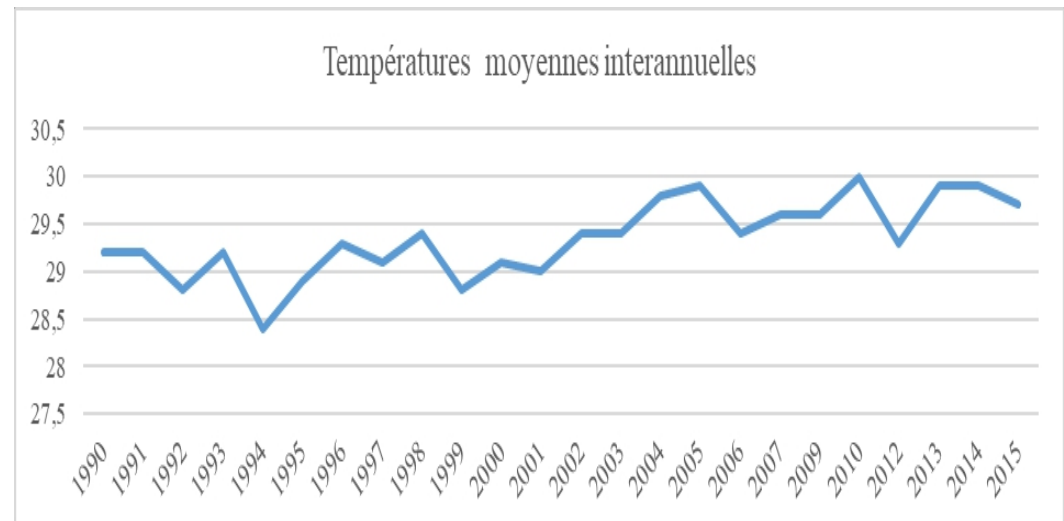

Figure 6 : évolution des températures moyennes interannuelles enregistrées à la station météorologique de San, de 1990 à 2015

Source : Météo-Mali, 2015

L'analyse de la courbe d'évolution des températures moyennes interannuelles montre une tendance à la hausse de façon générale dans la station de San. L'année 1994 est considérée comme l'année où la plus faible température fut enregistrée ces vingt-cinq (25) dernières années avec une moyenne de $28,4^{\circ} \mathrm{C}$. Depuis lors, la tendance est à la hausse jusqu'à atteindre une moyenne annuelle de $30^{\circ} \mathrm{C}$ en 2010 , soit un taux d'augmentation de $1,6^{\circ} \mathrm{C}$. Ce qui prouve que l'augmentation des températures évoquée par le Groupe d'Experts Intergouvernemental sur l'Evolution du Climat (GIEC) est bien une réalité dans notre zone d'étude.

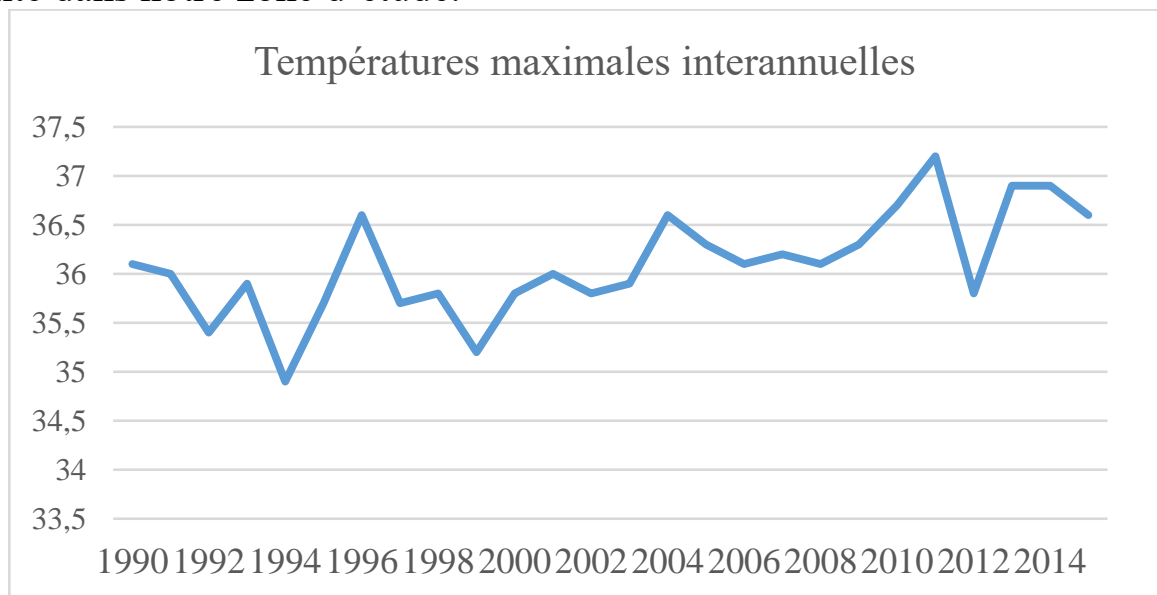

Figure 7 : évolution des températures maximales interannuelles enregistrées à la station météorologique de San, de 1990 à 2015

Source : Météo-Mali, 2015 
L'analyse de la courbe d'évolution des températures montre une tendance générale à la hausse. De 1990 à 1994, l'analyse de la courbe montre une tendance à la baisse. Toutefois, depuis 1994, la courbe est caractérisée par une augmentation de la température allant jusqu'à une moyenne maximale interannuelle de $37,2^{\circ} \mathrm{C}$ en 2010 considérée comme l'année la plus chaude. Une augmentation de $1,4^{\circ} \mathrm{C}$ des températures maximales a été enregistrée au cours des vingt-cinq (25) dernières années.

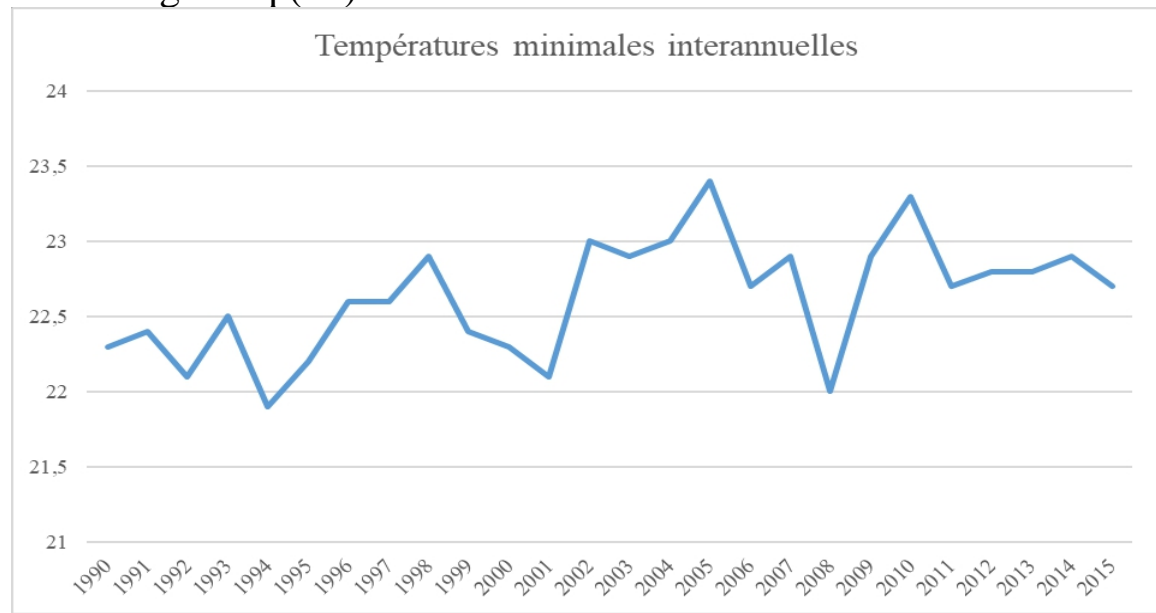

Figure 8 : évolution des températures minimales interannuelles enregistrées à la station météorologique de San, de 1990 à 2015

Source : Météo-Mali, 2015

L'analyse de la courbe d'évolution des températures minimales observées ces vingt-cinq (25) dernières années montre une tendance à la hausse. On constate une baisse remarquable de la température en 1994 allant jusqu'en dessous de $22^{\circ} \mathrm{C}$. Ainsi, dès lors, on observe une augmentation jusqu'à atteindre un taux record de $23,4^{\circ} \mathrm{C}$ en 2005 , soit l'année la plus chaude, considérée ces vingt-cinq (25) dernières années. Ensuite, la température a encore baissé en 2008 jusqu'à $22^{\circ} \mathrm{C}$ pour ensuite monter en 2010 . Au cours de ces vingt-cinq (25) dernières années, il a été enregistré une augmentation de $1,4^{\circ} \mathrm{C}$ dans les températures minimales. L'analyse des figures 7 et 8 montre une augmentation globale des températures observées entre 1990 et 2015 . Ce qui constitue d'ailleurs l'une des manifestations des changements climatiques. En ce qui concerne la pluviométrie, l'analyse des données statistiques des précipitations montre une diminution globale au niveau du Cercle. Mais, au niveau de la Commune Rurale de Mafouné, on constate une variabilité interannuelle très marquée. 


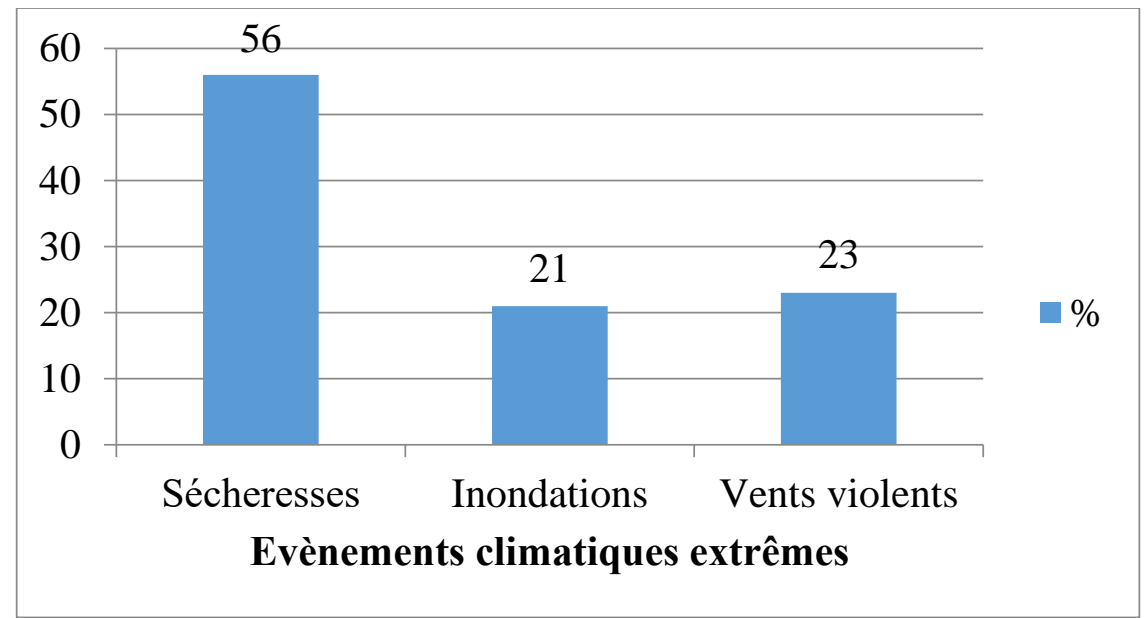

Figure 9 : perception paysanne sur les événements climatiques extrêmes

Les résultats de l'enquête montrent que les exploitants enquêtés perçoivent les événements climatiques extrêmes. Parmi les évènements cités, $56 \%$ des enquêtés ont évoqué la fréquence des sécheresses, contre $23 \%$ qui ont parlé des vents violents. Les inondations ont été évoquées par $21 \%$ des enquêtés. Ces différents évènements attestent des manifestations des effets des changements climatiques dans la Commune Rurale de Mafouné (photo 1).

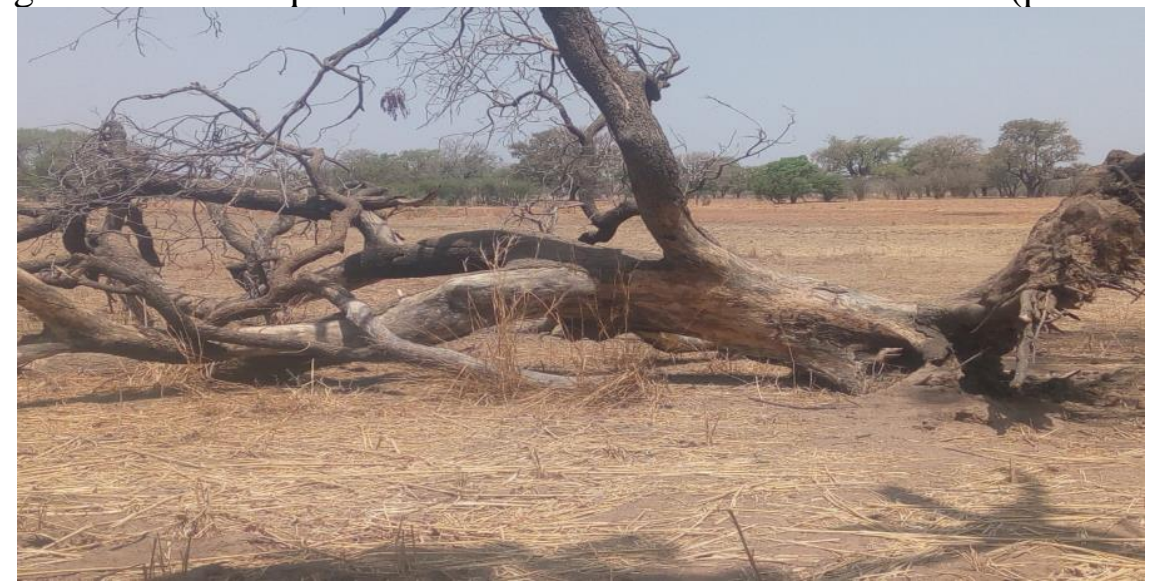

Photo 1 : tronc d'arbre de néré (Parkia biglobosa), arraché par un vent violent pendant l'hivernage 2017 à Mafouné

Ce gros tronc de néré arraché illustre l'effet des vents violents sur l'environnement, l'une des manifestations des changements climatiques à Mafouné. Cependant, ces vents violents deviennent de plus en plus fréquents pendant ces dernières années selon nos personnes enquêtées.

Les résultats obtenus sur l'analyse des perceptions des exploitants des changements climatiques montrent que le climat a globalement changé dans la Commune Rurale de Mafouné. Dans cette Commune, les changements 
climatiques se manifestent par des saisons de pluies de plus en plus marquées par la sécheresse, l'augmentation globale de la température et les évènements climatiques extrêmes. Les résultats de l'étude sont confirmés par les données météorologiques. En ce qui concerne la température, les modèles climatiques convergent pour montrer que notre zone d'étude est marquée par une augmentation des températures. Par rapport aux précipitations, les résultats de l'analyse montrent par contre, une variabilité pluviométrique interannuelle très marquée dans la Commune (figure 3). L'analyse de ces données a été faite sur les dix dernières années, faute de données suffisantes disponibles. Ce qui constitue une faiblesse, mais nous donne néanmoins une idée sur la situation pluviométrique de la zone. Pour mieux apprécier la situation climatique actuelle, nous avons également analysé les données pluviométriques recueillies sur plus de vingt-cinq ans au niveau du Cercle. Ces résultats ont montré une tendance pluviométrique à la baisse (figure 4) confirmant ainsi que la zone est sujette aux fluctuations climatiques.

Les résultats obtenus ont conclu sur l'augmentation des températures et la baisse de la pluviométrie, sans oublier leurs corolaires de vents violents, de poches de sécheresse et d'inondations. Ces résultats convergent vers ceux obtenus par Ballo et al. (2018) qui, ont signalé l'installation tardive de l'hivernage, la fin précoce de l'hivernage, la fréquence des poches de sécheresse, la mauvaise répartition des pluies, l'augmentation en intensité de la chaleur dans le cadre de leur étude sur les Communes Rurales de Sorobasso et Koumbri au Mali. Nos résultats sont corroborés par ceux de N'diaye (2015) qui rapporte dans ses études que, les paysans du Cercle de Banamba dans la Région de Koulikoro au Mali perçoivent les changements climatiques directement par la modification des paramètres pluviométriques, de températures et de vents. Nos résultats sont également confirmés par ceux obtenus par l'Agence de l'Environnement et du Développement Durable (AEDD) du Mali dans le rapport final d'élaboration de la Politique Nationale sur les Changements Climatiques en 2011. Cette Agence a trouvé que depuis l'apparition des périodes de sécheresse en 1970, on observe l'instauration d'un climat de plus en plus aride sur l'ensemble du territoire malien et un déplacement des isohyètes de $200 \mathrm{~km}$ vers le Sud.

\section{Impacts des changements climatiques sur les activités agricoles dans la Commune Rurale de Mafouné}

Les impacts des changements climatiques sur les activités agricoles dans la Commune Rurale de Mafouné sont à la fois environnementaux et socio-économiques. 


\section{Impacts environnementaux}

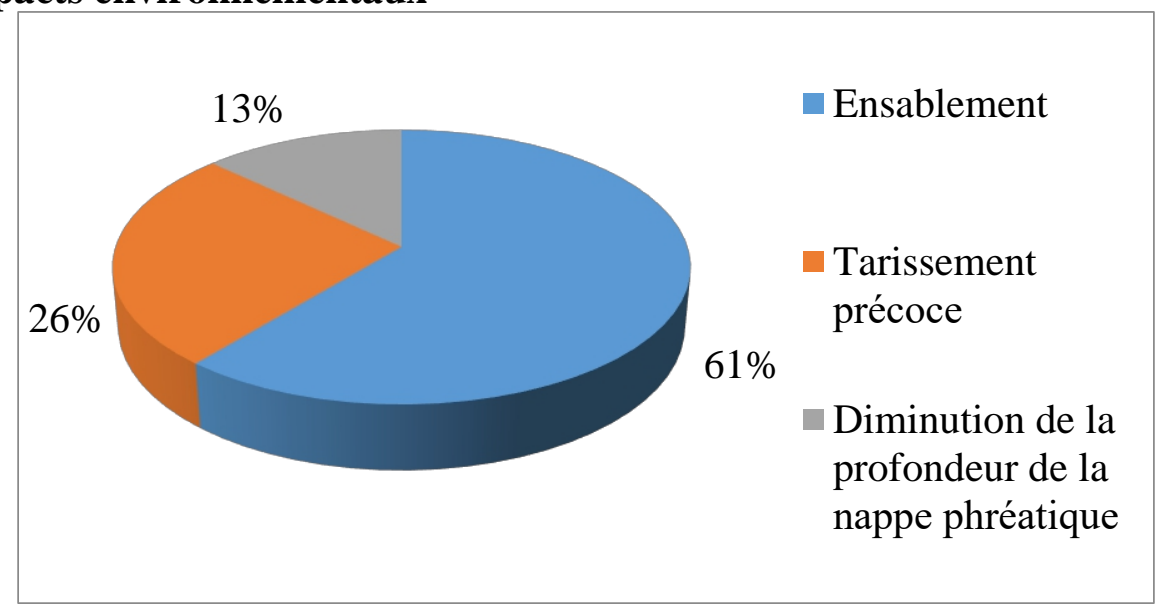

Figure 10 : impacts des changements climatiques sur les ressources en eau

L'analyse de la figure 10 montre que $61 \%$ des exploitants percoivent l'ensablement des cours d'eau et des mares, contre $26 \%$ qui observent le tarissement précoce des ressources en eau de surface. La diminution de la profondeur de la nappe phréatique a été évoquée par $13 \%$ des enquêtés.

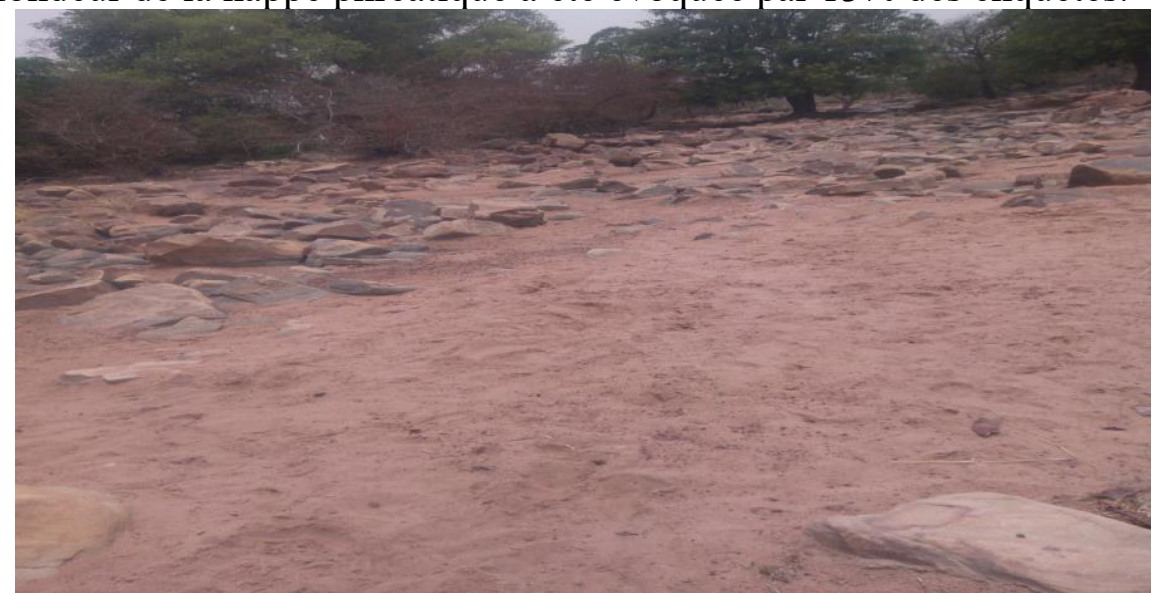

Photo 2 : cours d'eau en voie d'ensablement dans le village de Mouni dans la Commune Rurale de Mafouné

Cette photo montre l'ensablement d'un cours d'eau dans la Commune Rurale de Mafouné. Ce phénomène qui accentue le déficit hydrique serait lié aux changements climatiques. 


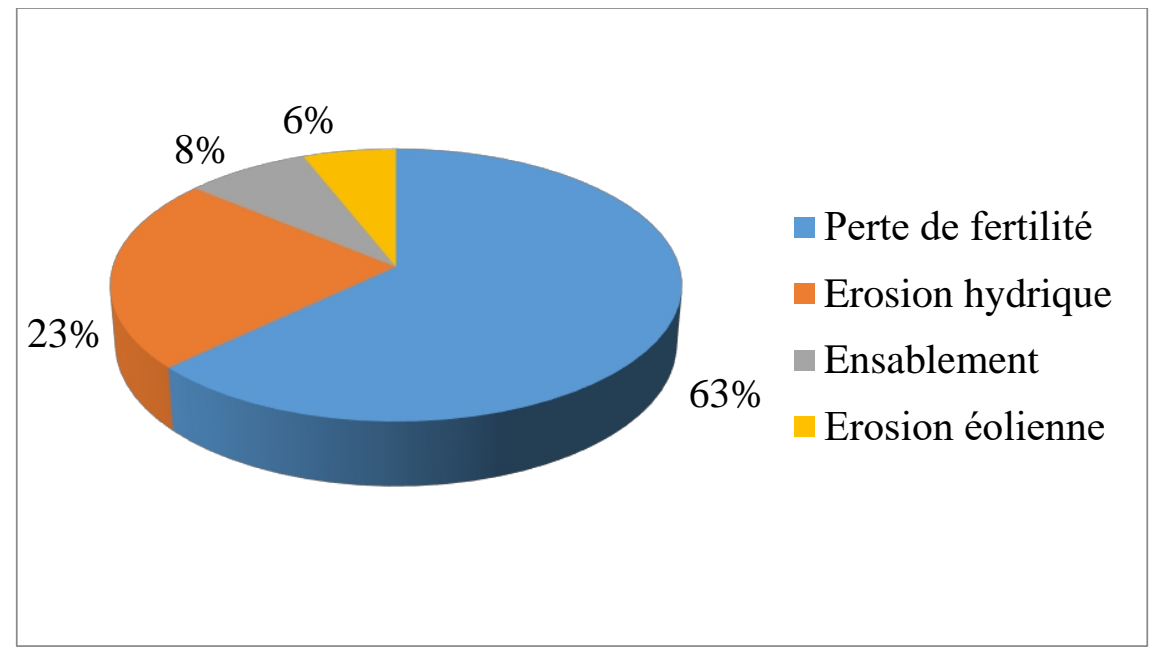

Figure 11 : impacts des changements climatiques sur les sols

La figure 11 montre que les principaux impacts des changements climatiques sur les sols perçus par les enquêtés sont : la perte de fertilité, l'érosion hydrique, l'ensablement et l'érosion éolienne. La majorité des enquêtés a évoqué la perte de fertilité, contre $23 \%$ qui ont parlé de l'érosion hydrique. L'ensablement et l'érosion éolienne ont respectivement été évoqués par $8 \%$ et $6 \%$ des enquêtés.

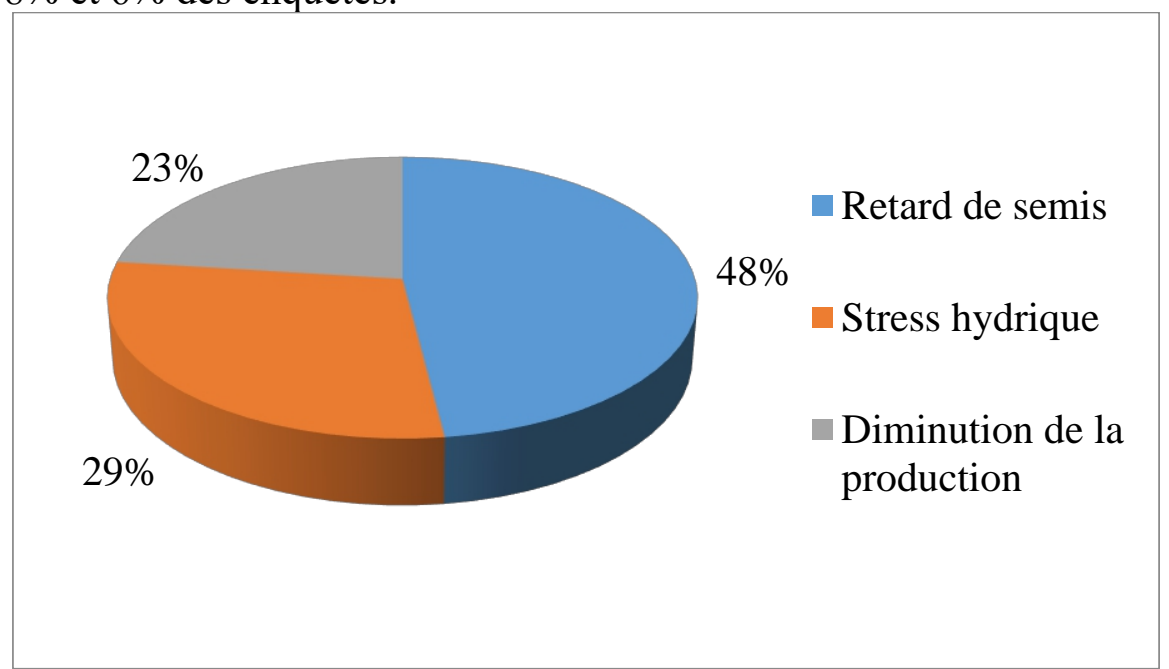

Figure 12 : impacts des changements climatiques sur les cultures

Les impacts des changements climatiques sur les cultures citées par les enquêtés vont du retard de semis, à la diminution de la production, en passant par le stress hydrique. Ces phénomènes qui affectent les rendements agricoles selon les enquêtés, s'expliqueraient par la baisse de la pluviométrie et sa mauvaise répartition spatio-temporelle. 


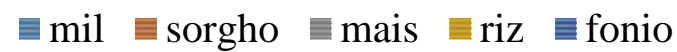

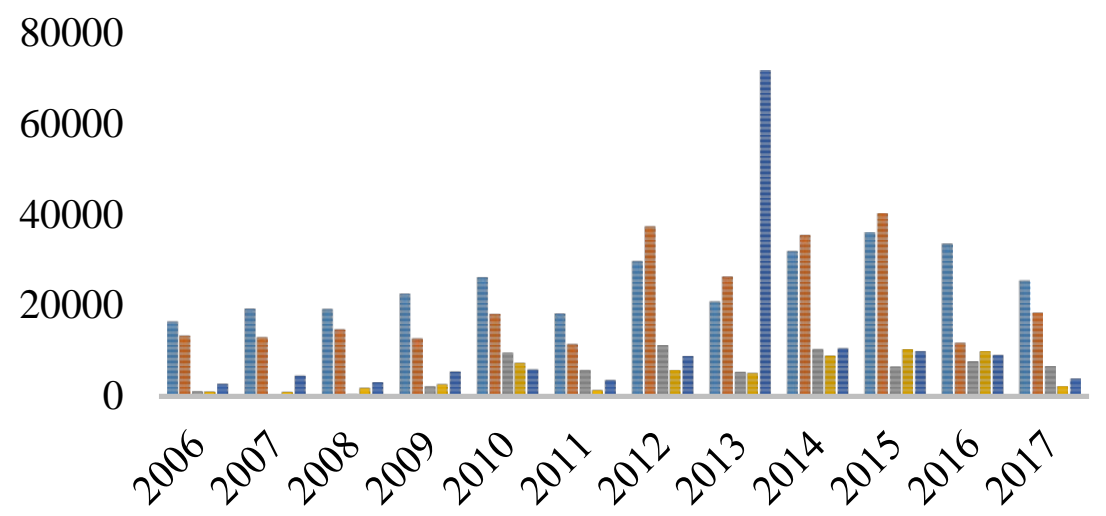

Figure 13 : évolution des productions céréalières dans le Cercle de Tominian, de 2006 à 2017

Source : Secteur Agriculture de Tominian, 2017

L'observation de la figure 13 permet de constater de façon générale une hausse des productions agricoles. Cette hausse est très remarquable pour les années 2012, 2013, 2014 et 2015 où environ plus de 30000 tonnes de mil et de sorgho ont été produites. Toutefois, à partir de 2015, on constate une légère diminution des productions malgré une pluviométrie assez suffisante. Ces hausses et ces diminutions tiendraient aux variations de la pluviométrie et sa répartition dans le temps et dans l'espace.

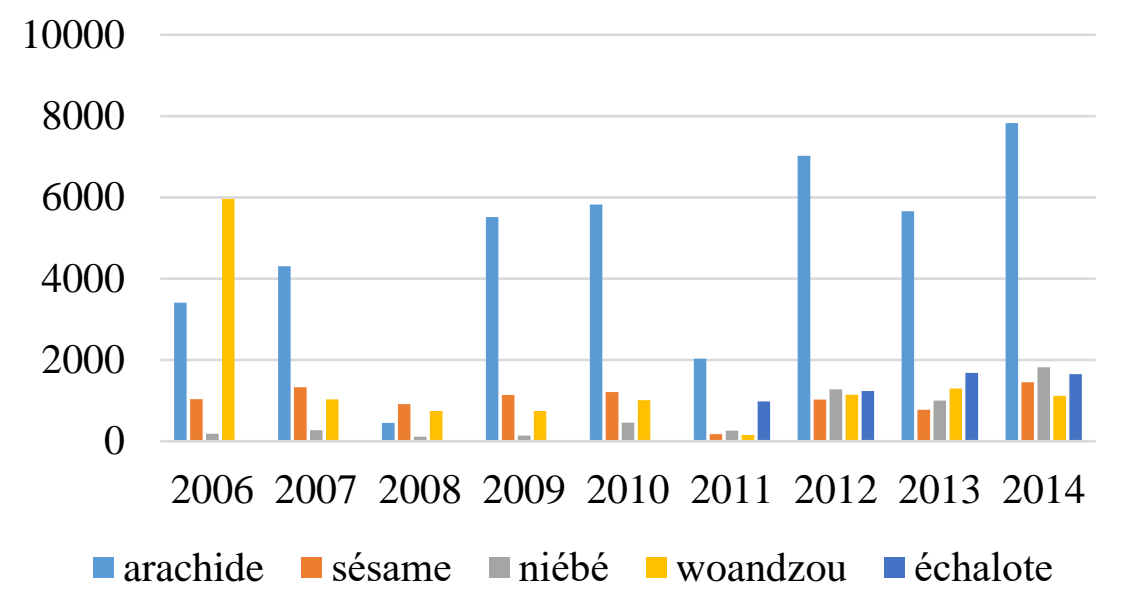

Figure 14 : productions des cultures légumineuses dans le Cercle de Tominian, de 2006 à 2014

Source : Secteur Agriculture de Tominian, 2014

En ce qui concerne la production des légumineuses, l'analyse de la figure 14 montre que l'arachide prend le dessus en termes d'importance avec 
un record d'environ 8000 tonnes produites en 2014. Pour les autres cultures, la production est presque stable et parfois même à la baisse, surtout pour les productions de 2011.

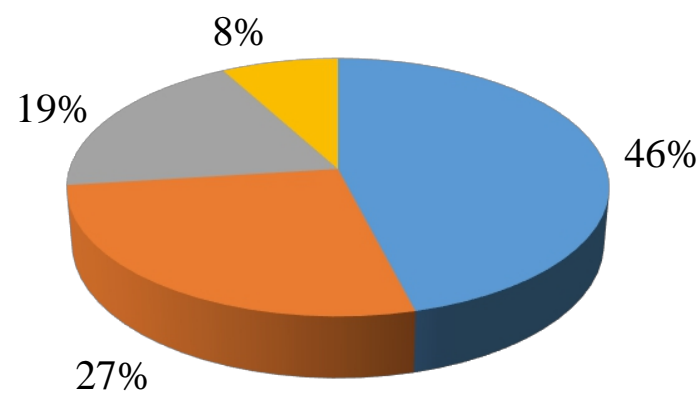

Diminution du couvert végétal

Diminution du peuplement ligneux et herbacé

- Mauvaise qualité fourragère

Figure 15 : impacts des changements climatiques sur la végétation et les ressources animales

Les impacts des changements climatiques perçus par les populations sur la végétation et les ressources animales sont la diminution du couvert végétal, la diminution du peuplement ligneux et herbacé, la mauvaise qualité fourragère et le manque de fourrage.

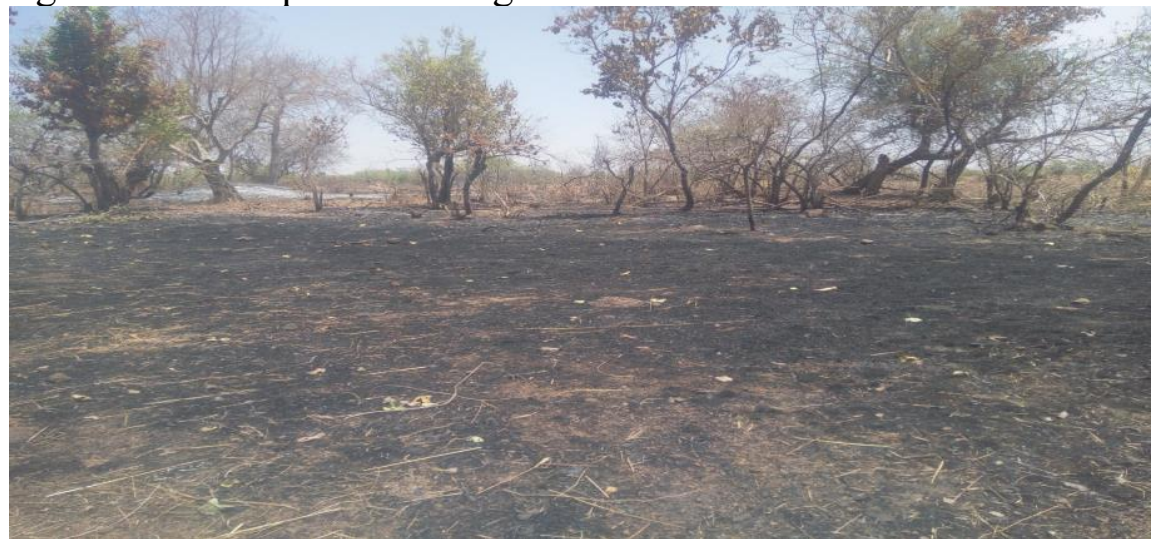

Photo 3 : forêt dévastée par un feu de brousse à Mafouné

La photo 3 illustre la destruction du couvert végétal et de l'habitat de la faune dans la Commune Rurale de Mafouné.

\section{Impacts socio-économiques}

Les impacts socio-économiques des changements climatiques cités par les exploitants agricoles de la zone d'étude sont indiqués dans le tableau 1 cidessous. 
Tableau 1 : impacts socio-économiques des changements climatiques

\begin{tabular}{lc}
\hline Impacts socio-économiques & Réponses (en \%) \\
\hline $\begin{array}{l}\text { Baisse des rendements, insécurité } \\
\text { alimentaire }\end{array}$ & 49 \\
Diminution des revenus, endettement & 22 \\
Accentuation de la pauvreté & 16 \\
Intensification de l'exode rural & 13 \\
Total & $\mathbf{1 0 0}$ \\
\hline
\end{tabular}

L'analyse du tableau 1 montre que près de la majorité des enquêtés (49\%) perçoivent la baisse des rendements et l'insécurité alimentaire comme impacts socio-économiques des changements climatiques. La diminution des revenus et l'endettement ont été évoqués par 22\% des enquêtés, contre $16 \%$ qui perçoivent l'accentuation de la pauvreté. L'intensification de l'exode rural a été rapportée par 13\% des exploitants enquêtés.

Les résultats de l'étude montrent que les impacts des changements climatiques perçus par les exploitants agricoles de la Commune Rurale de Mafouné, sont à la fois environnementaux et socio-économiques. Ils portent essentiellement sur le tarissement précoce des ressources en eau, la réduction de la disponibilité de l'eau (stress hydrique), l'érosion hydrique, la perte de fertilité des sols, le retard des semis, la baisse des rendements, le raccourcissement de la durée de la saison pluvieuse, la réduction du couvert végétal, la diminution des revenus des exploitants, l'insécurité alimentaire, l'accentuation de la pauvreté et l'intensification de l'exode rural dans la Commune. La perception des impacts des changements climatiques sur les activités agricoles dans la Commune Rurale de Mafouné dans l'ensemble est proche des résultats de l'analyse des données des services d'agriculture (figures 13 et 14). Nos résultats sont similaires à ceux obtenus par le Programme d'Action National d'Adaptation aux Changements Climatiques (PANA, 2007), qui a montré que la diminution de la pluviométrie perçue par les paysans a entrainé le tarissement des eaux de surface et la baisse du niveau de la nappe phréatique. En plus de la baisse de la fertilité, la zone d'étude connait une forte dégradation de son couvert végétal selon la perception des enquêtés. Ces deux phénomènes ont accentué la perte des potentialités productives comme l'a confirmé le PANA (2007), qui faisait état de la perte de la fertilité des sols et de la réduction du taux de matières organiques liés aux effets des conditions climatiques défavorables. Nos résultats vont également dans le même sens que ceux de Bagayoko et al. (2010), qui avaient montré la baisse de la production céréalière dans le cadre de leur étude sur les changements climatiques sur les cultures céréalièères dans le Cercle de Bougouni au Mali. Les résultats obtenus par notre étude sont confirmés par ceux du GIEC dans son rapport de 2007. Ce rapport indiquait que les changements climatiques vont modifier le régime des précipitations, 
augmenter la fréquence des chocs climatiques et induire des transformations des écosystèmes. La pauvreté et l'insécurité alimentaire soulignées par les enquêtés sont des facteurs déjà considérés par le Comité Inter-Etats de Lutte contre la Sécheresse au Sahel (CILSS). Cette organisation confirme que la fragilisation des ressources en eau, la baisse des rendements agricoles, la prévalence accrue des maladies des cultures, conjuguées à une forte augmentation du coût de l'énergie et des denrées alimentaires risqueraient d'accentuer l'insécurité alimentaire et la pauvreté à l'horizon 2020 (CILSS, 2009).

\section{Stratégies d'adaptation aux changements climatiques}

Face aux manifestations des changements climatiques, les exploitants agricoles de la Commune Rurale de Mafouné développent des stratégies d'adaptation. Ces stratégies nous ont été rapportées lors des focus group et sont présentées dans le tableau 2 qui suit.

Tableau 2 : stratégies d'adaptation des enquêtés aux changements climatiques

\begin{tabular}{|c|c|}
\hline Impacts des changements climatiques & Stratégies d'adaptation \\
\hline $\begin{array}{l}\text { Mauvaise installation des pluies, retard } \\
\text { de semis }\end{array}$ & $\begin{array}{l}\text { Ressemis, semis sec, semis des variétés } \\
\text { à cycle court, abandon des cultures de } \\
\text { rente }\end{array}$ \\
\hline Erosion hydrique, pauvreté des sols & $\begin{array}{c}\text { Demi-lunes, cordons pierreux, } \\
\text { billonnage, association des engrais } \\
\text { organiques et minéraux }\end{array}$ \\
\hline Diminution du couvert végétal & $\begin{array}{l}\text { Reboisement, Régénération Naturelle } \\
\text { Assistée, agro-foresterie, lutte contre les } \\
\text { feux de brousse }\end{array}$ \\
\hline $\begin{array}{l}\text { Diminution des rendements, baisse des } \\
\text { revenus, pauvreté, endettement, exode } \\
\text { rural }\end{array}$ & $\begin{array}{l}\text { Diversification des activités, (petit } \\
\text { commerce, maraîchage, élevage) }\end{array}$ \\
\hline
\end{tabular}

Le tableau 2 montre que les exploitants enquêtés développent plusieurs stratégies pour s'adapter aux effets néfastes des changements climatiques. Ainsi, en cas de mauvaise installation des pluies et de retard des semis, les exploitants ont recours au ressemis et au semis sec. Aussi, ils utilisent les variétés hâtives, ou abandonnent les cultures de rente, et plus particulièrement, le coton. Pour lutter contre l'érosion hydrique, les exploitants emploient la technique des demi-lunes, les cordons pierreux et le billonnage pour éviter le ruissellement de l'eau. Face à la pauvreté des sols, ils emploient massivement des engrais à la fois organiques et minéraux. Pour lutter contre la diminution du couvert végétal, l'analyse des résultats montre que le reboisement, la Régénération Naturelle Assistée (RNA), l'agro-foresterie, la lutte contre les feux de brousse, sont des pratiques majoritairement adoptées par les exploitants. La diversification des activités à travers la pratique du petit commerce, le maraîchage, l'élevage, sont des stratégies développées par les 
exploitants pour faire face à la diminution des rendements agricoles, la baisse des revenus, la pauvreté, l'endettement et l'exode rural. Les photos 4, 5 et 6 ci-dessous, illustrent quelques pratiques d'adaptation développées par les exploitants agricoles de la zone d'étude.

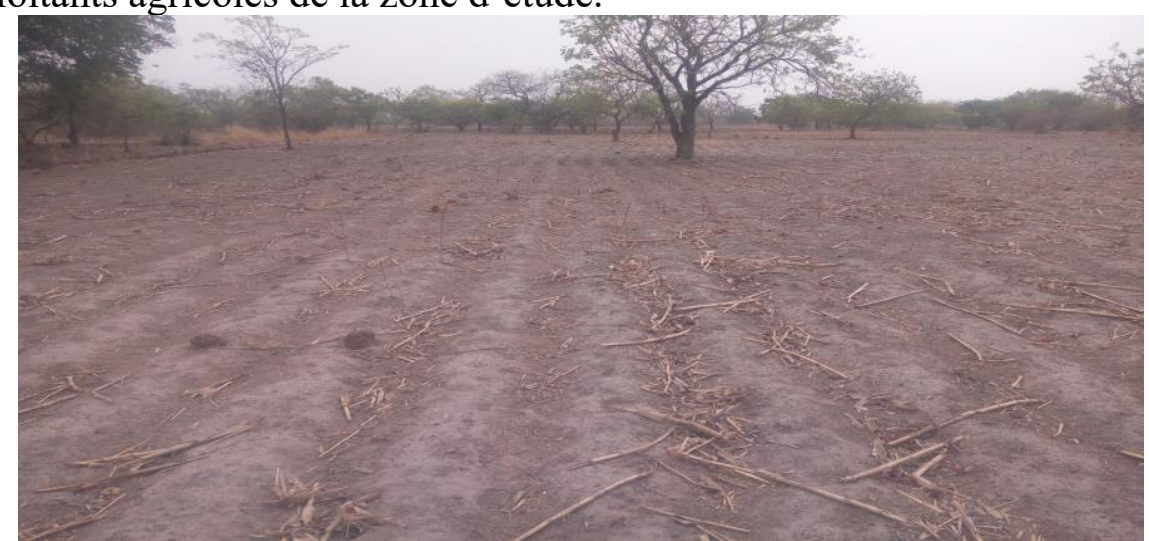

Photo 4 : pratiques d'adaptation contre l'érosion hydrique à Mafouné

La photo 4 montre un champ de billons. La technique du billonnage est utilisée contre l'érosion hydrique. Elle favorise l'infiltration des eaux de pluie et maintient une fraîcheur dans le champ. Cette technique est majoritairement utilisée par les exploitants agricoles de la Commune Rurale de Mafouné.

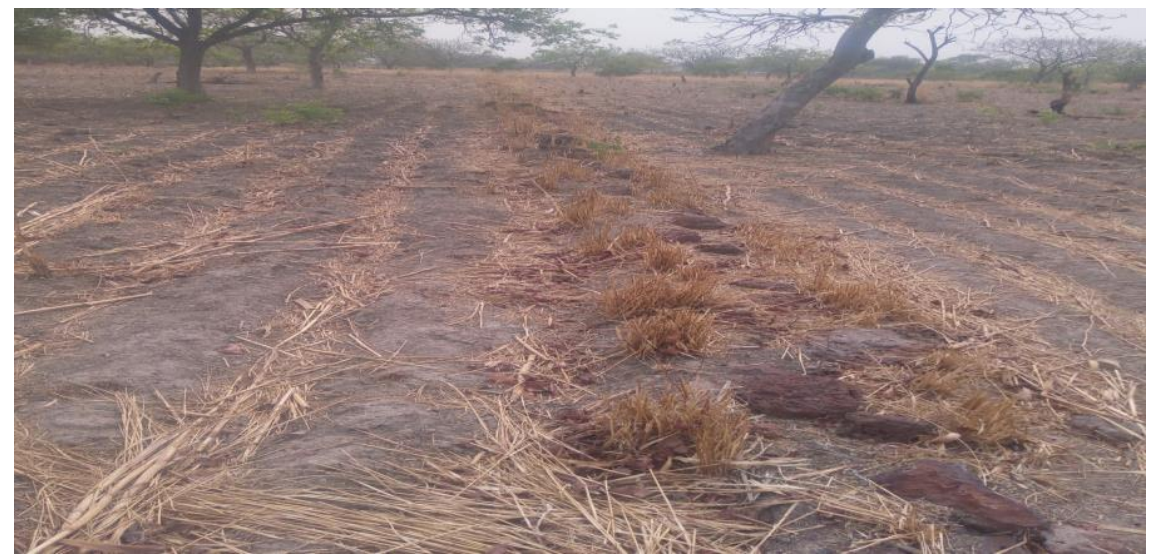

Photo 5 : réduction de l'érosion hydrique par les cordons pierreux à Mafouné

La photo 5 illustre la technique des cordons pierreux dans un champ pour réduire l'érosion hydrique ainsi que le ruissellement des eaux pluviales, généralement dans le sens contraire de la pente. Ceci permet de récupérer les sols, mais aussi, de stocker des éléments drainés par les eaux de ruissellement sous forme de fumier, très favorable à la croissance des plantes. Cette technique est majoritairement utilisée par les paysans, mais, elle exige la 
mobilisation d'énormes moyens notamment, une main-d'œuvre nombreuse pour le ramassage et le transport des cailloux, parfois très éloignés du champ.

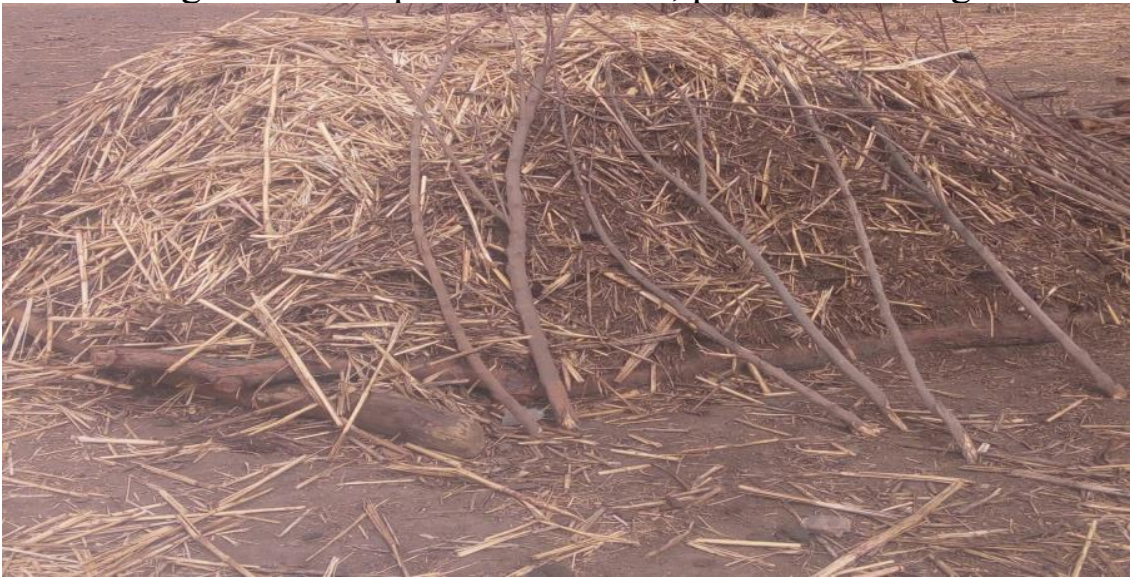

Photo 6 : fabrication du fumier organique à Mafouné

La photo 6 montre l'image du fumier en phase de fabrication à partir des débris de tiges de mil mélangés avec de la terre qui sera apporté dans les champs. Ceci confirme en effet la pauvreté des sols évoquée par les enquêtés.

Les résultats de ce travail montrent que les exploitants agricoles de la Commune Rurale de Mafouné développent plusieurs stratégies pour faire face aux impacts des changements climatiques. Ces stratégies d'adaptation portent notamment sur les ressemis, les semis des variétés à cycle court, l'abandon des cultures de rente, l'utilisation de la technique des demi-lunes, des cordons pierreux, le billonnage, l'association des engrais organiques et minéraux, Reboisement, Régénération Naturelle Assistée (RNA), l'agro-foresterie, la lutte contre les feux de brousse, la diversification des activités. Il est important de souligner que ces différentes stratégies d'adaptation pratiquées par les exploitants sont pour la plupart l'œuvre des différentes Organisations Non Gouvernementales et des projets qui interviennent dans la Commune Rurale de Mafouné. Les personnes enquêtées dans leur majorité (85\%) affirment avoir appris ou ont été sensibilisées par des agents des projets ou des ONG sur ces techniques d'adaptation qui, d'ailleurs sont bénéfiques pour la protection de leur environnement et la conservation des sols. Les stratégies d'adaptation évoquées par nos enquêtés convergent vers celles obtenues par Ballo et al. (2018), qui rapportent que la diversification des activités, la reprise des semis, les interdictions de couper les arbres sur les berges, l'élevage de petits ruminants, le maraîchage, l'utilisation des intrants, des variétés précoces et résistantes, constituent les stratégies d'adaptation des exploitants des Communes Rurales de Sorobasso et Koumbri dans le Cercle de Koutiala au Mali. Ces pratiques d'adaptation doivent reposer sur une bonne maîtrise. C'est pourquoi, elles nécessitent aujourd'hui un accompagnement permanent des 
exploitants pour leur permettre de mieux répondre aux impacts des changements climatiques sur les activités agricoles et de rehausser les productions agricoles dans la Commune Rurale de Mafouné en particulier et au Mali en général.

\section{Conclusion}

La présente étude est une contribution à la connaissance des perceptions des exploitants agricoles des impacts des changements climatiques sur les activités agricoles dans la Commune Rurale de Mafouné et les stratégies d'adaptation en réponse aux effets induits par les changements climatiques.

En dépit de la faiblesse de la taille de l'échantillon enquêté, de l'insuffisance des données climatologiques sur de longues années, nous pouvons dire que la mauvaise répartition des pluies, le retard dans le démarrage de la saison pluvieuse, les poches de sécheresse au cours de la saison pluvieuse, les inondations, les vents violents, sont des éléments qui caractérisent le climat ces dernières décennies dans la zone d'étude. Les différents impacts des changements climatiques perçus par les exploitants agricoles et les pratiques d'adaptation adoptées montrent clairement que les activités agricoles subissent lourdement les effets néfastes des changements climatiques. Face à cette situation d'incertitude climatique, un besoin en appui technique pour mieux adapter les activités agricoles à la situation climatique actuelle s'impose dans la Commune Rurale de Mafouné. Un tel appui permettrait de mieux structurer les pratiques locales développées par les populations elles-mêmes. La baisse de la pluviométrie, l'extrême variabilité, l'augmentation de la température accentuant l'évapotranspiration et le tarissement précoce des eaux de surface, la destruction des écosystèmes très fragilisés par la désertification, la forte pression démographique sur les ressources naturelles, amènent à s'interroger sur l'avenir des populations de la Commune, qui tirent l'essentiel de leurs moyens de subsistance des ressources naturelles. Face à ces aléas climatiques, il y va de l'intérêt de tous d'orienter la réflexion vers un nouveau paradigme et de comprendre que les changements climatiques sont aujourd'hui une réalité. Cela devrait taire le débat sur l'existence des changements climatiques et interpeler l'opinion publique internationale que le moment n'est plus aux discours, mais, plutôt aux actes.

Ce travail n'est pas exhaustif. Par conséquent, des études plus approfondies permettraient de prendre en compte d'autres aspects de la question afin d'aider les populations rurales à mieux faire face aux changements climatiques. 


\section{References:}

1. AGRIDAPE. (2009). Changements climatiques, entre résilience et résistance, volume $24, \mathrm{~N}^{\circ} 4$, UNESCO, Paris.

2. Bagayoko, A.B., Hien E. (2010). Impacts des changements climatiques sur les cultures céréalières dans le Cercle de Bougouni.

3. Ballo, M., Bengaly A., Sidibé A. (2018). Perceptions paysannes de la manifestation des changements climatiques sur l'exploitation des basfonds dans le Cercle de Koutiala: cas de la Commune Rurale de Sorobasso et Koumbri (Région de Sikasso-Mali), in Revue Africaine des Sciences Sociales et de la Santé Publique (RASP), №17, juilletdécembre 2018, pp.125-149.

4. CILSS. (2009). Changement climatique et risque pour la sécurité alimentaire et la biodiversité en Afrique : Expérience du CILSS au Sahel et en Afrique de l'Ouest. Accessible sur URL : http://publications.insah.org, consulté le 14 février 2018.

5. CCNUCC. (2015). Rapport de la $21^{\text {ème }}$ Conférence des Parties (COP21), Paris.

6. GIEC. (2007). Bilan 2007 des changements climatiques, rapport de synthèse.

7. GIEC. (2014). Incidences, adaptation et vulnérabilité : résumé à l'intention des décideurs, Contribution du groupe de travail II au cinquième rapport d'évaluation $\mathrm{du}$ Groupe d'Experts Intergouvernemental sur l'Evolution du Climat.

8. Konaté, F.O. (2009). Perceptions et stratégies d'adaptation des populations de la Commune Rurale de Nossombougou aux changements climatiques (Cercle de Kolokani, Région de Koulikoro au Mali), in Recherches africaines, $\mathrm{N}^{\circ} 09$. Accessible sur URL : http://www.recherches-africaines.net, consulté le 16 février 2018.

9. Mairie de la Commune Rurale de Mafouné. (2011). Plan de Prévention et de Gestion des Catastrophes (PPGC) de la commune rurale de Mafouné, rapport final.

10. Mairie de la Commune Rurale de Mafouné. (2011). Programme de Développement Economique, Social et Culturel-PDESC, 2016-2020 de la Commune Rurale de Mafouné.

11. Ministère de l'Environnement et de l'Assainissement et Agence de l'Environnement et du Développement Durable. (2011). Politique Nationale sur les Changements Climatiques (PNCC) du Mali.

12. Ministère de l'Environnement et de l'Assainissement du Mali. (2011). Stratégie Nationale du Changement climatique (SNCC), rapport final.

13. Ministère de l'Equipement et des Transports et Direction Nationale de la Météorologie. (2007). Programme d'Action National d'Adaptation aux Changements Climatiques (PANA). Bamako, Mali. 
14. N'diaye, B.F. (2015). Changements climatiques et dynamiques des systèmes de production agricole dans le Cercle de Banamba, Région de Koulikoro au Mali, thèse de doctorat, Institut Supérieur de Formation et de Recherche Appliquée (ISFRA). Bamako, Mali.

15. Sanogo, M.K. (2012). Capitalisation des bonnes pratiques de gestion durable des terres pour l'adaptation à la variabilité et au changement climatique au Mali: analyse d'impacts agronomiques, environnementaux et socio-économiques, mémoire de Master, Centre Régional AGRHYMET. Niamey, Niger. 